\title{
Leonhard Euler als Theoretiker
}

\section{Einleitung}

Der irische Satiriker Jonathan Swift hatte gesagt: „Elephanten werden stets kleiner als in Wirklichkeit gezeichnet, aber ein Floh stets größer" (Fellmann 2007, S. XIII). Wer immer über Euler etwas sagen will, muss genau dieses Problem lösen: Wie wird man einem Mathematiker gerecht, der gleichsam ein Shakespeare der Mathematik war: universell, reichhaltig in den Einzelheiten, unerschöpflich (Simmons 2007, S. 168), der alle Gebiete der reinen und angewandten Mathematik behandelte und bereicherte, dessen mathematisches, philosophisches und religiöses Denken einander bedingten?

Tatsächlich war Euler tief religiös, ein überzeugter reformierter Protestant. 1744 erschien seine Theorie der Bewegungen der Planeten und Kometen in Berlin bei Ambrosius Haude (Euler 1744a). Ferdinand Helfreich Frisch gab dem Werk den folgenden Kupferstich bei (Abb. 1: EO II, 28, Tafel I vor S. 105; Fellmann 2007, S. X). Er verdeutlicht Eulers Vorstellung von Gottes Schöpfung. Zahllose Sonnensysteme werden von Sonnen, Planeten, Monden, Kometen gebildet. Noch ist der erst im Jahre 1781 von Friedrich Wilhelm Herschel entdeckte Uranos nicht berücksichtigt. Die Schöpfung ist das Werk des Weisesten, sapientissimi opus, wie der Spruch besagt. Aber der Mathematiker kann durch Anwendung des Newtonschen Gravitationsgesetzes und Aufstellung von Differentialgleichungen die Bahnen berechnen, das heißt aus der Ursache, der Kraft, die Wirkung, die Bahn. Davon zeugt der Kupferstich zu Beginn der 1736 erschienenen Mechanik oder analytisch dargelegte Wissenschaft von der Bewegung (Abb. 2: Euler 1736, S. 5).

Das Haupt der Himmelsgöttin trägt die Sonne, um die die mit bloßen Augen erkennbaren sechs Planeten mit einigen ihrer Monde kreisen. In der rechten Hand hält die Göttin ein aufgeschlagenes Buch. Die Zeichnungen zeigen die elliptische Bahn eines Planeten in deren Gänze oder zu einem Teil.

Für den gläubigen Euler gab es noch einen zweiten Weg zur Welterkenntnis. Gottes Schöpfung ist höchst vollkommen und unterliegt deshalb Extremalprinzipien. Im 

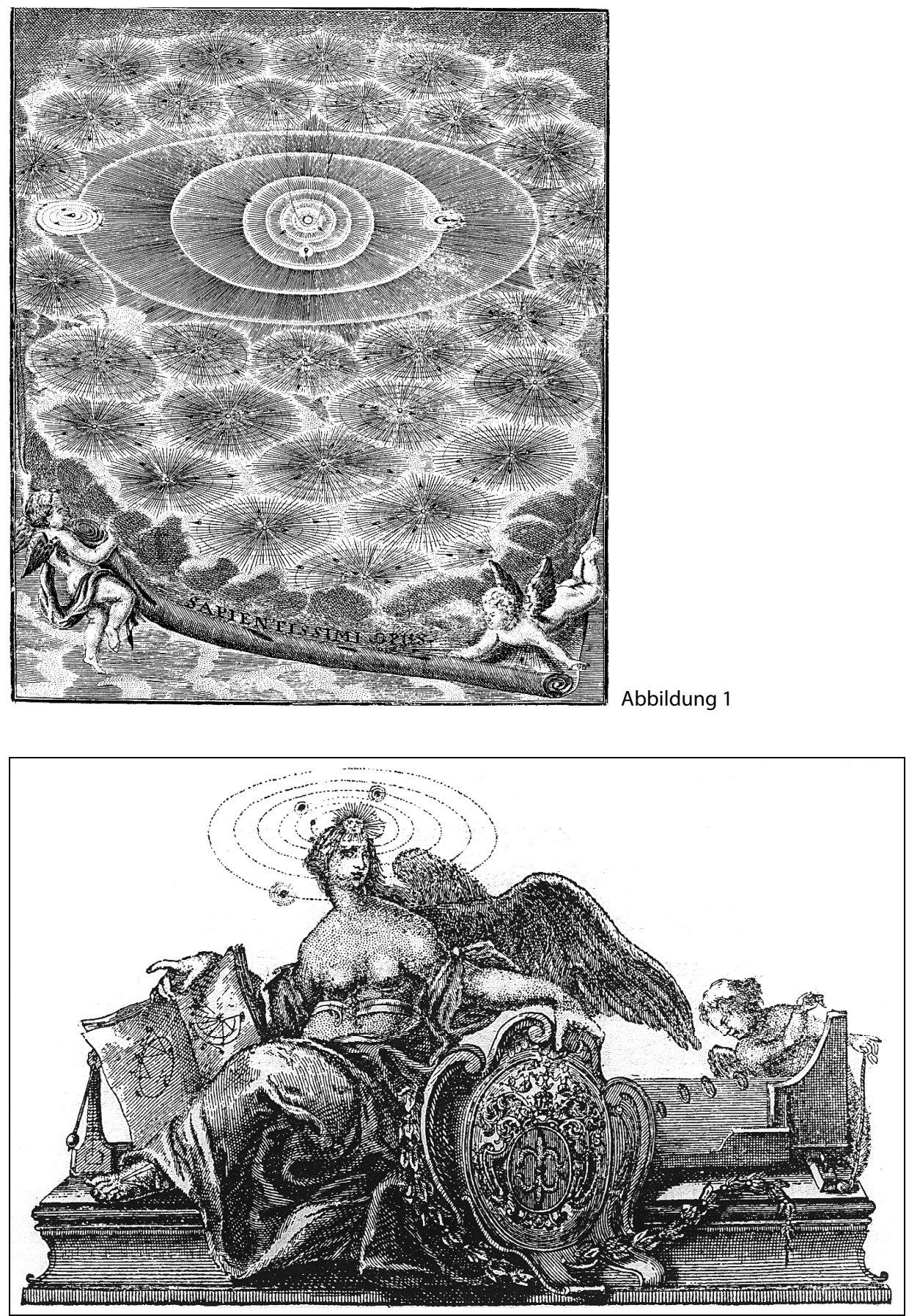

Abbildung 2

242 Eberhard Knobloch 
1. Zusatz zu seiner 1744 veröffentlichten Variationsrechnung gab Euler folgende gern zitierte Erklärung ab (Euler 1744b, S. 231; Thiele 1982, S. 69; Szabó 1987, S. 105): „Cum enim mundi universi fabrica sit perfectissima atque a Creatore sapientissimo absoluta, nihil omnino in mundo contingit, in quo non maximi minimive ratio quaepiam eluceat; quamobrem dubium prorsus est nullum, quin omnes mundi effectus ex causis finalibus ope methodi maximorum et minimorum aeque feliciter determinari queant, atque ex ipsis causis efficientibus.“ „Da nämlich der Bau der gesamten Welt höchst vollkommen und vom weisesten Schöpfer vollendet wurde, geschieht überhaupt nichts in der Welt, bei dem nicht das Verhältnis eines Maximums oder Minimums hervorleuchtet; deshalb gibt es durchaus keinen Zweifel, dass alle Wirkungen der Welt, die auf Finalursachen beruhen, mit Hilfe der Methode der Maxima und Minima gleich erfolgreich bestimmt werden können wie aus den bewirkten Ursachen selbst.“

Die Worte verdeutlichen, wie eng Eulers Philosophie, Religiosität und Mathematik miteinander zusammenhingen, wie stark diese einander wechselseitig bedingten: Der creator sapientissimus tritt in der Planetentheorie ebenso wie in der Variationsrechnung auf. Ich möchte deshalb die folgenden vier Aspekte des Theoretikers Euler ansprechen:

I Philosophisches Denken

II Theologisches Denken

III Über das Unendliche

IV Zur Musiktheorie

Den Schluss wird ein kurzer Epilog bilden.

\section{Philosophisches Denken}

Euler hat sich wiederholt energisch und polemisch zu philosophischen Fragen geäußert, und zwar um die Leibniz-Wolffsche Philosophie zu bekämpfen bzw. das, was er dafür hielt. Besondere Bedeutung erlangten drei Auseinandersetzungen.

1. Die 1745 für 1747 unter Eulers Einfluss von der Berliner Akademie gestellte Preisaufgabe (EO III, 2, S. XI):

„On demande, qu'en commençant par exposer d'une manière exacte et nette la doctrine des Monades, on examine si d'un côté elles peuvent être solidement réfutées et détruites par des argumens sans réplique; ou si de l'autre on est en état, après avoir prouvés les Monades, d'en déduire une explication intelligible des principaux phénomènes de l'Univers, et en particulier de l'origine et du mouvement des corps". 
Auf Betreiben Eulers wurde der Preis dem Monadengegner Johann Heinrich Gottlob von Justi 1747 zuerkannt.

2. Die Debatte um das Prinzip der kleinsten Aktion und den Schweizer Mathematiker Samuel König in den Jahren 1751 bis 1753, die zum Eingreifen des Königs Friedrich II. und Voltaires führte und den Akademiepräsidenten Maupertuis zum Verlassen Berlins trieb (Knobloch 1995).

3. Die 1753 für 1755 von der Berliner Akademie gestellte Preisaufgabe:

„L'examen du système de Pope (Tout est bien)““

Der Preis wurde auf Drängen Maupertuis' dem Antileibnizianer A. F. Reinhard zuerkannt.

Warum war Euler derart heftig gegen die Leibniz-Wolffsche Philosophie eingestellt? Das Kernstück der Leibnizschen Philosophie war die Lehre von den Monaden, dem Nicht-Materiellen jedes Lebewesens, den menschlichen Seelen, denen weder Ausdehnung noch Gestalt zukommt (Finster \& van den Heuvel 1990, S. 65). Während Materie nach Leibniz unendlich teilbar ist, haben Monaden als individuelle Substanzen keine Teile. Jeder Monade ist aber ein materieller Körper zugeordnet. Die Beziehung zwischen Monade und ihrem Körper ist durch eine prästabilierte, von Gott geschaffene Harmonie geregelt.

Soweit Leibniz. Euler machte den Unterschied zwischen Substanz, sprich Monaden, und den zugeordneten Körpern nicht mit. Er redete, wie Breidert prägnant sagte, dogmatisch an den Dogmatikern vorbei (Breidert 1983, S. 451). Die Leibnizsche Lösung des Leib-Seele-Problems, die prästabilierte Harmonie, lehnte er von Anbeginn ab, so bereits in seiner Habilitationsschrift über den Schall (Euler 1727, S. 196). In den Briefen an eine deutsche Prinzessin hatte er nur Spott dafür übrig: Sollte sein Körper nicht weiter mit seiner Seele zusammentreffen, so würde er ihm nicht mehr angehören als der Körper eines Rhinoceros in Afrika. „Ich würde“, schreibt er im Brief 83, „eben soviel als jetzt, die Ehre haben, Ew. H. zu schreiben; aber wie sie alsdann meine Briefe aufnehmen würden, das weiß ich nicht.“ (Euler 1768-1772, S. 188).

Leibniz war seit langem tot. Eulers furor wandte sich gegen die Monadisten, das heißt gegen den Hallenser Rationalisten und Aufklärungsphilosophen Christian Wolff und dessen Anhänger. Er unterstellte ihnen atheistische Tendenzen, ein Punkt, bei dem er keinen $\mathrm{Spa} ß$ verstand.

Als die Berliner Akademie 1745 die Preisaufgabe stellte, die Monadenlehre exakt darzustellen und gegebenenfalls daraus Ursprung und Bewegung der Körper abzuleiten, ließ Euler 1746 anonym die Schrift Gedancken von den Elementen der Cörper, in welchen das Lehr-Gebäude von den einfachen Dingen und Monaden geprüfet, und das wahre Wesen der Cörper entdecket wird erscheinen (Euler 1746a; dazu Matter \& Burckhardt in EO III, 2, S. XI): Eulers Autorschaft war freilich nicht zu verheimlichen. Eine Kampfschrift gegen die ungeliebten Vertreter der Monadenlehre. Euler 
unterstellte diesen, sie ließen die Körper aus einfachen Dingen, den Monaden, bestehen. Dem setzte er seine Lehre entgegen, dass Körper qua Ausdehnung ohne Grenze ins Unendliche teilbar sind, dass Ausdehnungsloses nichts ist, dass sie also nicht aus einfachen Dingen, den Monaden zusammengesetzt sein können: Eulers Widerlegung der Monadologie, die für erheblichen Widerspruch bei den angegriffenen Philosophen gesorgt hat, zumal der Preis einem Monadengegner zuerkannt wurde. In den Briefen an eine deutsche Prinzessin gab Euler diesem Thema nochmals breiten Raum (Brief 122-132). Ironisch bemerkte er (Brief 125; Euler 1986, S. 144; Euler 1768 1772, III, 11, S. 295): „Herr von Wolf selbst, [...] bedrohte schon unsere Akademie mit dem philosophischen Bannstrahle. Ich erinnere mich nicht mehr, wem wir es eigentlich zu danken haben, daß wir ihm noch entgangen sind“".

Eulers Streitschrift erschien mit einem Emblem: Über der Büste der behelmten Göttin Athene beziehungsweise Minerva, der Göttin der Künste und der Weisheit, prangt der Hexameterschluss eines Verses von Horaz (Epistulae I, 2, 40): Sapere aude, „Habe Mut, Deinen Verstand zu gebrauchen.“

Kant, der zu Euler Kontakt gesucht hatte, hatte diese Aufforderung 1784 zum Wahlspruch der Aufklärung in seiner Beantwortung der Frage: Was ist Aufklärung? erhoben (Kant 1784, S. 51). Schiller griff den Imperativ 1794 im 8. Brief Über die ästhetische Erziehung des Menschen auf (Schiller 1794, S. 591).

Man könnte darin einen zusätzlichen Seitenhieb Eulers auf die beschränkten Monadisten sehen. Doch tritt das Motto auch zum Beispiel auf den Titelblättern der Theorie der Bewegungen der Planeten und Kometen von 1744 und der Neuen Grundsätze der Artillerie von Robins auf, von denen Euler 1745 eine deutsche Bearbeitung veröffentlichte (Euler 1745): Es ist das Emblem des Verlegers Johann Ambrosius Haude, der ab 1744 das Recht besaß, alle Veröffentlichungen der Akademie der Wissenschaften zu verlegen. Er starb jedoch bereits vier Jahre später.

Mit welcher Unbekümmertheit Euler naturphilosophische Fragen behandelte, mag seine 1746 erschienene Aus mechanischen Prinzipien abgeleitete Entwirrung der Frage, ob der Materie die Fähigkeit zu denken zugeteilt werden kann oder nicht verdeutlichen (Euler 1746b). Euler bediente sich eines Syllogismus:

Kein Körper hat eine zur Trägheit gegenteilige Kraft.

Die Fähigkeit zu denken ist eine zur Trägheit gegenteilige Kraft.

Also kann kein Körper die Fähigkeit zu denken haben.

\section{Theologisches Denken}

In Glaubensfragen verstand Euler keinen Spaß. Wiederholt hat er seinen Glauben verteidigt, gegebenenfalls mit den Mitteln der Mathematik. Die zeitgenössische Philosophie Christian Wolffs, die Zweifler an der Wahrheit der biblischen Schöpfungs- 
geschichte, die Freigeister an der Berliner Akademie boten hinreichend Anlass zum Einschreiten.

1747 veröffentlichte er die kleine, kaum zwanzig Seiten umfassende Schrift Rettung der göttlichen Offenbarung gegen die Einwürfe der Freygeister (Euler 1747). Schwierigkeiten und scheinbare Widersprüche können weder die Geometrie noch die Heilige Schrift in Misskredit bringen. Die Auferstehung Christi ist danach eine ausgemachte Wahrheit (§36), die Existenz von Teufeln sehr wahrscheinlich: „Da allem Anschein nach Gott von allen möglichen Arten Geschöpfe erschaffen hat, so haben wir nicht den geringsten Grund an dem Daseyn solcher uns an Verstand eben so weit als an Boßheit übertreffenden Geschöpfe zu zweifeln“ (Euler 1747, S. 272). In den Briefen an eine deutsche Prinzessin führt er seine Gedanken zur göttlichen Vorsehung fort. Die Menschen sind weder die besten noch die bösesten Geister, die wir Teufel nennen (Brief 111). Ganz ähnlich argumentiert Kant in der etwa zeitgleich erschienenen Allgemeinen Naturgeschichte und Theorie des Himmels (Kant 1755, S. 387f.). Sein Sonnenabstandsgesetz besagte, dass die Wesen um so moralisch höher stehend und klüger sind, auf je entfernteren Planeten sie wohnen. Ein aktuelles Thema, wenn man an die jüngste Entdeckung des bewohnbaren Planeten V 838 Monoceratis in 200 Billionen km Entfernung denkt.

Dementsprechend meinte Euler, der Schöpfungsplan erforderte wohl, dass alle möglichen Geister existieren (Brief 110). Eulers Teufels-, nicht Gottesbeweis erforderte eine Theodizee: Hätte Gott nicht besser diejenigen Geister nicht erschaffen, von denen er vorhersah, dass sie ihre Freiheit missbrauchen und sündigen werden? Ironisch antwortet Euler auf solche Einwürfe: „Gewiß würden diejenigen, die Gott diesen Vorwurf machen, sehr ungern zur Ausnahme gehören.“

Zudem gilt (Brief 111): „Jeder Geist, und selbst der Teufel nicht ausgenommen, ist immer ein herrliches Wesen (un être excellent), das unendliche Vorzüge vor all dem hat, was man sich in der körperlichen Welt nur denken kann“ (Euler 1768-1772, III, 11, S. 264).

Der Prinzessin vermittelt Euler (Brief 113): Die Trübsale dieser Welt sind notwendig. Gott hat nicht ohne die stärksten Gründe soviel Elend und Trübsale in diese Welt eingeführt.

In der Einführung in die Analysis des Unendlichen dient die Berechnung des Bevölkerungswachstums als Beispiel für das Rechnen mit Logarithmen (Euler 1748 I, S. 115f.): Nach der Sintflut sei das Menschengeschlecht von 6 Menschen fortgepflanzt worden. Wenn es nach 200 Jahren auf 1 Million angewachsen ist, hätte es jährlich um den 16. Teil von sich wachsen müssen. Wenn sich in den einzelnen Jahrhunderten die Zahl der Menschen verdoppeln soll, muss sich die Zahl jährlich um den 144. Teil vermehren, was offensichtlich kein Problem ist. „Quam ob causam maxime ridiculae sunt eorum incredulorum hominum obiectiones, qui negant tam 
brevi temporis spatio ab uno homine universam terram incolis impleri potuisse," bemerkte Euler (Euler 1748, I, S. 116), „Deshalb sind die Einwände derjenigen ungläubigen Menschen äußerst lächerlich, die sagen, in so kurzem Zeitraum hätte die gesamte Erde nicht von einem Menschen mit Einwohnern angefüllt werden können.“ Entsprechende Rechnungen hat „Professor Euler“, wie der Geistliche Johann Peter Süßmilch dankbar in seinem Werk über das Bevölkerungswachstum vermerkt, diesem 1761 zur Verfügung gestellt (Süssmilch 1761, S. 513) und in weiteren Veröffentlichungen wie den Recherches générales sur la mortalité et la multiplication du genre humain (Euler 1767) angestellt. Eulers Verdienste um die Bevölkerungsstatistik wurden in Berlin dadurch gewürdigt, dass das eine der beiden großen Reliefs am Statistischen Bundesamt in Berlin-Mitte auch sein Profil (neben dem von Leibniz, Newton, Einstein) enthält (Hoffmann 1992, S. 47f.). Allenthalben ist Gottes Schöpfung gemäß der biblischen Schöpfungsgeschichte in Eulers Denken gegenwärtig. Die Schöpfung lag ja nach dessen Überzeugung kaum 6000 Jahre zurück. Das Licht des hinter der Sonne nächsten Fixsterns brauchte nach Eulers Berechnung sechs Jahre. Wenn die Sterne zur gleichen Zeit wie Adam erschaffen worden wären, so hätte dieser sie nicht eher als nach sechs Jahren sehen können, selbst nicht den nächsten. Wenn also Gott noch tausendmal entferntere Sterne erschaffen hätte, so würden wir sie noch nicht sehen, so glänzend sie auch sein möchten, weil noch nicht 6.000 Jahre verflossen sind, erklärte Euler der Prinzessin (Brief 20).

In der ersten Arbeit zur Ableitung der ersten Lösung eines Spezialfalles (kollinearer Fall) des Dreikörperproblems bewunderte Euler die Weisheit Gottes als Schöpfer der Welt. Wäre der Mond hundertmal entfernter, müsste er sich wie ein Hauptplanet bewegen. Wäre die Entfernung zehnmal größer, wären wir im Zweifel, ob er ein Planet oder ein Mond ist. Aber der weiseste Schöpfer hat mit Rücksicht auf unseren unzureichenden Verstand keinen Himmelskörper so platziert (Euler 1766a, S. 248).

\section{III Über das Unendliche}

Das Unendliche spielte in Eulers Denken eine überragende Rolle: die Analysis des Unendlichen erlaubte ihm, seine großartigen Leistungen in der Hydromechanik wie in der Himmelsmechanik. Wie aber ging er damit um? Drei Beispiele mögen seinen Umgang mit dem Unendlichen veranschaulichen.

\section{Das Basler Problem}

Euler hat das Prinzip angewendet, das Nils Henrik Abel zum ersten Mal in Worte gekleidet hat (Engel/Schlesinger 1913, S. XI): Probleme so fassen, dass sie lösbar werden. Ein zunächst unlösbares Problem war die Summierung der reziproken 
Quadratzahlen. Jakob Bernoulli erkannte die Konvergenz der Reihe, da diese die konvergente Majorante der reziproken Dreieckszahlen hat. Aber er klagte über die Schwierigkeit der Aufgabe und sagte 1689 (Stäckel 1925, S. 160): „Sollte jemand das, was unseren Anstrengungen bis jetzt entgangen ist, finden und uns mitteilen, so werden wir ihm sehr dankbar sein.“

Euler fand den Wert

$$
\frac{\pi^{2}}{6}
$$

im Herbst 1735 und teilte ihn seinem Freund Daniel Bernoulli mit (Stäckel 1925, S. 162; Hofmann 1959, S. 185).

Die Algebra war, wie Condorcet in seiner Eloge auf Euler sagte (Condorcet 1786, S. 290), eine „science très-bornée“. Euler sprengte diese Grenzen. Eine Potenzreihe ist ein ad infinitum fortgesetztes Polynom. Man darf mit ihr wie mit Polynomen rechnen. Die Analysis der transzendenten Funktionen ist nichts anderes als eine natürliche Erweiterung der Algebra. Die Faktorzerlegung von Polynomen kann auf transzendente Funktionen, das heißt Gleichungen unendlich hohen Grades, angewandt werden (Euler 1748, Kap. 10). Danach gilt (Polya 1954, S. 41-46; Simmons 2007, S. 267-269):

a) $\sin x=\frac{x}{1}-\frac{x^{3}}{3 !}+\frac{x^{5}}{5 !}-\frac{x^{7}}{7 !} \pm \ldots=0$

hat die unendlich vielen Wurzeln

$0, \pm \pi, \pm 2 \pi, \pm 3 \pi, \ldots$

b) Division durch $\mathrm{x}$ ergibt:

$$
\frac{\sin x}{x}=1-\frac{x^{2}}{3 !}+\frac{x^{4}}{5 !}-\frac{x^{6}}{7 !} \pm \ldots=0
$$

Diese ,unendliche Gleichung“ hat die Wurzeln $\pm \pi, \pm 2 \pi, \pm 3 \pi, \ldots$

c) Die Zerlegung in Faktoren eines Polynoms geraden Grades $2 n$ mit den $2 n$ verschiedenen Wurzeln $\pm a_{1}, \pm a_{2}, \ldots, \pm a_{n}\left(a_{i} \neq 0\right)$ :

$$
b_{0}-b_{1} x^{2}+b_{2} x^{4} \ldots+(-1)^{n} b_{n} x^{2 n}=b_{0}\left(1-\frac{x^{2}}{a_{1}{ }^{2}}\right)\left(1-\frac{x^{2}}{a_{2}{ }^{2}}\right) \ldots\left(1-\frac{x^{2}}{a_{n}{ }^{2}}\right)
$$

Nach den Girard-Newton-Leibniz-Formeln zu Gleichungskoeffizienten und Gleichungswurzeln gilt:

$$
b_{1}=b_{0}\left(\frac{1}{a_{1}^{2}}+\frac{1}{a_{2}^{2}}+\ldots+\frac{1}{a_{n}^{2}}\right)
$$


d) Analogie: Schritt von endlicher zu unendlicher Gleichung

$$
\begin{aligned}
& \frac{\sin x}{x}=\left(1-\frac{x^{2}}{\pi^{2}}\right)\left(1-\frac{x^{2}}{4 \pi^{2}}\right)\left(1-\frac{x^{2}}{9 \pi^{2}}\right) \ldots \\
& \text { nun ist } b_{0}=1, b_{1}=\frac{1}{3 !}, \\
& \text { also } \frac{1}{3 !}=\frac{1}{\pi^{2}}+\frac{1}{4 \pi^{2}}+\frac{1}{9 \pi^{2}}+\ldots \\
& \text { oder } \frac{\pi^{2}}{6}=1+\frac{1}{4}+\frac{1}{9}+\ldots
\end{aligned}
$$

Euler schreibt zu dem Zeitpunkt

a) noch $\mathrm{p}$ statt $\pi$ (Euler 1740). Sein Verfahren erlaubte ihm

b) die Berechnung der Terme der Potenzen $1+\frac{1}{2^{n}}+\frac{1}{3^{n}}+\ldots$

für gerades $n$. Seine 2. Veröffentlichung zum Thema erschien

c) bereits in Berlin (Euler 1743). 1743 schreibt er: „methodus [...] utique erat nova et in eiusmodi instituto plane non usitata; nitebatur enim in resolutione aequationis infinitae, cuius omnes radices, quarum numerus erat infinitus, nosse oportebat", „,die Methode war [...] jedenfalls neu und bei einem derartigen Unternehmen völlig ungebräuchlich; sie stützte sich nämlich auf die Lösung einer unendlichen Gleichung, deren gesamte Wurzeln, deren Zahl unendlich groß war, man kennen musste.“

Er glaubte eine Zeitlang, ein anderer Weg ohne die Lösung einer unendlichen Gleichung stünde nicht offen. Doch er fand andere Lösungen. In diesem Berliner Aufsatz von 1743 führte er $\pi$ für den halben Umfang eines Kreises mit Radius 1 ein.

d) Euler wandte eine Regel auf einen Fall an, für den sie nicht gemacht war: statt einer algebraischen Gleichung nahm er eine nicht-algebraische Gleichung: ein Analogieschluss.

Aber er hatte Gründe, dem Ergebnis zu trauen:

a) die numerische Übereinstimmung mit unmittelbarer Berechnung der Summe endlich vieler Terme

b) die Ableitung der Leibnizschen Reihe für $\frac{\pi}{4}$. 


\section{Divergente Reihen}

Im Jahre 1754/55 verfasste Euler seinen Aufsatz De seriebus divergentibus, der 1760 erschien. Zunächst definiert er die Begriffe konvergente-divergente Reihe.

Def. 1 Konvergent heißen bei ihm Reihen, deren Terme eine streng monotone Nullfolge bilden, wie

$$
1+\frac{1}{2}+\frac{1}{4}+\ldots
$$

Die Reihe besteht aus termini continuo decrescentes. Dies ist nicht der moderne Konvergenzbegriff, denn die harmonische Reihe

$$
1+\frac{1}{2}+\frac{1}{3}+\ldots
$$

wäre danach konvergent, während sie nach modernem Verständnis divergent ist, das heißt ohne endlichen Summenwert: Die Folge der Partialsummen übersteigt jeden endlichen Wert. Euler führt nur eine notwendige Bedingung an.

Def. 2 Divergent heißen Reihen, deren Terme endlich bleiben oder ins Unendliche wachsen.

Euler sagt: termini infinitesimi non in nihilum abeunt, das heißt: Die infinitesimalen Terme werden nicht zu nichts. Euler führt nach heutigem Verständnis nur eine hinreichende Bedingung für Divergenz an. Sie ist nicht notwendig, da auch die harmonische Reihe im heutigen Sinn divergiert.

Fazit: Eulers und der moderne Konvergenz- beziehungsweise Divergenzbegriff unterscheiden sich. Euler definiert mit Hilfe der Größe der Terme. Heute definiert man mit Hilfe des Grenzwertes der Folge der Partialsummen.

Wir müssen akzeptieren: Euler hatte einen anderen Konvergenzbegriff als wir heute. Zu Recht stellten Engel und Schlesinger 1913 fest (Engel \& Schlesinger 1913, S. XIIf.), dass eine Abschätzung des formalen Prozesses fehlt, um einen vorgeschriebenen Genauigkeitsgrad zu erzielen. Auch wenn es Euler im Differentialkalkül anders darstellt: die Strenge der Griechen, und das heißt von Archimedes, erreicht er nicht (Euler 1755, S. 71). Wir kommen darauf zurück.

Ausgangspunkt war die alternierende Reihe $1-1+1-1+1 \ldots$

Am 10. Januar 1714 teilte Leibniz Johann Bernoulli, dem späteren Lehrer Eulers, das nach ihm benannte Konvergenzkriterium für alternierende Reihen mit:

Alternierende Reihen sind konvergent, wenn die Folge der Reihenglieder monoton abnehmend gegen Null konvergiert (Leibniz 1856, S. 926). Seine Mitteilung stand im Zusammenhang mit einem Brief an Christian Wolff aus dem Jahre 1713 (Leibniz 1713). 
Er wusste, dass

$$
1-x+x^{2} \mp \ldots=\frac{1}{1+x}
$$

für jedes $\mathrm{x}$, das der Doppelungleichung $-1<\mathrm{x}<1$ genügt. Er setzte formal $\mathrm{x}=1$ ein und erhielt

$$
1-1+1-1 \ldots=\frac{1}{2} \text {. }
$$

Das Ergebnis hielt er für richtig, auch wenn darüber zwischen Guido Grandi, Wolff, Annibale Marchetti, Nikolaus I. und Daniel Bernoulli, Christian Goldbach gestritten wurde. Leibniz ging es um die Rechtfertigung. Diese fand er im Würfelspiel: Haben zwei Größen dieselbe Wahrscheinlichkeit, ist der mathematische Erwartungswert ihr arithmetisches Mittel, im vorliegenden Fall also

$$
\frac{0+1}{2}=\frac{1}{2} .
$$

Man kann dies als eine erste Idee von Summierbarkeit gemäß Cesàro oder Hölder sehen (Varadarajan 2006, S. 126). Grandi hatte eine Analogie zur Rechtsprechung im Falle zweier Erben vorgeschlagen, die von Leibniz zurückgewiesen worden war. Euler knüpfte an Leibnizens Brief an Wolff an (Euler 1760, 589). Er behandelte jedoch die alternierende Reihe von Leibniz sofort im allgemeinen Kontext der von ihm sogenannten divergenten Reihen, wie sie oben definiert wurden.

Er ging von Potenzreihenentwicklungen aus, wie sie bereits Leibniz betrachtet hatte:

$$
\begin{aligned}
& \frac{1}{1+x}=1-x+x^{2}-x^{3}+x^{4} \mp \ldots \\
& \text { bzw. } \frac{1}{1-x}=1+x+x^{2}+x^{3}+x^{4}+\ldots \\
& \text { oder } \frac{1}{(1-x)^{2}}=1+2 x+3 x^{2}+4 x^{3}+5 x^{4}+\ldots
\end{aligned}
$$

Setzt man formal - ohne Rücksicht auf den Konvergenzradius, modern gesprochen für x die Werte 1, 2, 3 usf. ein, erhält man die Gleichungen (Euler 1755, S. 81; Euler 1760, S. 591)

$$
\begin{aligned}
& \frac{1}{2}=1-1+1-1+1-1 \ldots \\
& \frac{1}{3}=1-2+4-8+16-32 \ldots
\end{aligned}
$$




$$
\begin{aligned}
& \frac{1}{4}=1-3+9-27+81-243 \ldots \\
& \text { bzw. } \frac{1}{0}=1+1+1+1+\ldots \\
& -1=1+2+4+8+\ldots \\
& -\frac{1}{2}=1+3+9+27+\ldots \\
& \text { bzw. } \frac{1}{0}=1+2+3+4 \ldots \\
& 1=1+4+12+32+\ldots
\end{aligned}
$$

Alle diese Reihen sind divergent im Eulerschen Sinn. Eulers Ziel war, den Nutzen solcher divergenten Reihen zu bewahren.

Die Frage war also: Lassen sich diese Gleichungen rechtfertigen?

a) $\frac{1}{2}$ und die alternierende Reihe $1-1+1-1 \pm \ldots$ sind jedenfalls quantitates aequivalentes (Euler 1760, S. 593). Die Frage bleibt, ob $\frac{1}{2}$ die Summe dieser Reihe genannt werden kann.

b) $\frac{1}{3}$ ist nicht die Summe der alternierenden Reihe $1-2+4-8 \ldots$ im Sinne einer schrittweisen Summierung, da sich bei schrittweiser Berechnung der Teilsummen die Ergebnisse immer weiter von $\frac{1}{3}$ entfernen.

c) Zum Ergebnis der Gleichungen (5), (6) heißt es: ,,multo minus cum solitis ideis conciliari potest“, ,Es kann noch viel weniger mit den gewohnten Ideen in Einklang gebracht werden.“

Sein gewagter Vorschlag lautete (Euler 1760, S. 592): „Interim tamen veritati consentaneum videtur, si dicamus easdem quantitates, quae sint nihilo minores, simul infinito maiores censeri posse.“ „Einstweilen scheint es indessen mit der Wahrheit übereinzustimmen, wenn wir sagen dieselben Größen, die kleiner als Null sind, zugleich als größer als Unendlich eingeschätzt werden können." Euler erklärt nicht, was für Größen dies sein könnten, die größer als Unendlich sind.

Für Euler reduzieren sich alle Schwierigkeiten auf den Begriff Summe. Dieser darf nicht operational verstanden werden (Euler 1755, S. 82; 1760, S. 593): „Dicamus ergo seriei cuiusque infinitae summam esse expressionem finitam, ex cuius evolutione illa series nascatur.“ „Wir wollen also sagen, die Summe jeder unendlichen Reihe ist der 
endliche Ausdruck, aus dessen Entwicklung jene Reihe entsteht.“ Diese Definition tritt wohl zum ersten Mal in einem Brief an Christian Goldbach vom 7.8.1745 auf (Faber 1935, S. XII). Euler hatte vor allem drei Rechtfertigungen für seine neue Summendefinition:

a) Permanenzprinzip

Die neue Definition fällt mit der alten zusammen, wenn es sich um konvergente Reihen handelt.

b) Eulers Transformation im Falle alternierender Reihen

Das Ergebnis stimmt mit dem überein, was seine Transformationsmethode lieferte:

Sei $\mathrm{s}=\mathrm{a}_{1}-\mathrm{a}_{2}+\mathrm{a}_{3}-\mathrm{a}_{4}+\mathrm{a}_{5}-\mathrm{a}_{6} \ldots$

Er berechnet die ersten, zweiten usf. Differenzen der aufeinander folgenden Reihenterme:

$$
\begin{aligned}
& \Delta a=a_{2}-a_{1}, a_{3}-a_{2}, a_{4}-a_{3}, \ldots \\
& \Delta^{2} a=a_{3}-2 a_{2}+a_{1}, a_{4}-2 a_{3}+a_{2}, \ldots \\
& S=\frac{1}{2} a_{1}-\frac{1}{4} \Delta a+\frac{1}{8} \Delta^{2} a \mp \ldots \\
& \Delta a=0,0,0, \ldots, a_{1}=1
\end{aligned}
$$

(Grattan-Guinness 1970, S. 68-70).

Also ist $S=\frac{1}{2}$.

Ebenso liefert die Methode für (2) $\mathrm{S}=\frac{1}{3}$, (3) $\mathrm{S}=\frac{1}{4}$ (Euler 1755, 224).

c) Die neue Definition erweist Eulers divergente Reihen als nützlich.

Euler kommentiert (Euler 1755, S. 82): „Ope huius definitionis utilitatem serierum divergentium tueri atque omnibus iniuriis vindicare poterimus.“ „Mit Hilfe dieser Definition werden wir die Nützlichkeit divergenter Reihen schützen und vor allen Ungerechtigkeiten bewahren können“".

Die Frage ist, ob Eulers Definition wohldefiniert ist. Dazu müsste er zeigen, dass es nur genau einen endlichen Ausdruck gibt, dessen Entwicklung zur betrachteten Reihe führt. Ein Gegenbeispiel gibt Faber (Faber 1935, S. XIII).

Für uns bleibt wichtig:

a) Euler verließ bewusst, wie im Falle des Basler Problems, gewohnte Ideen, ein Zeichen für Kreativität: Nimmt man nur Potenzreihen, so handelt es sich um das Abelsche Summierungsverfahren. 
$\sum a_{n}=f(1), f(x)=a_{0}+a_{1} x+\ldots$

erzeugende Funktion (Varadarajan 2006, S. 130).

b) Eine Analogie führte zu einer Regeldehnung, die durch eine Begriffsdehnung gerechtfertigt wird.

c) Die durch Analogie erhaltenen Ergebnisse wurden nicht bezweifelt, sondern es wurde nach einer Rechtfertigung gesucht. Euler fragte: Wie definiere ich, nicht: was ist?

d) Die Autoren unterschieden sich nur durch die Methoden der Rechtfertigung. Leibniz verwandte eine analoge Betrachtung, parallelisierte unendliche Reihen und Wahrscheinlichkeitstheorie. Euler wählte die Methode der Interpretation, der neuen Definition alter Begriffe. Dies war die Methode der englischen Mathematiker um John Peacock, Robert Woodhouse, Augustus de Morgan Anfang des 19. Jahrhunderts. Diese verwandten das Permanenzprinzip formaler Potenzreihen. Tatsächlich erhält Eulers Definition durch den Begriff der analytischen Fortsetzung von Karl Weierstrass einen bestimmten mathematischen Sinn (Faber 1935, S. XIIIf.).

\section{Die strenge Grundlegung der Differentialgleichung}

Der Differentialkalkül wurde wegen fehlender Strenge und verdächtiger Schlussfolgerungen zu Eulers Zeiten kritisiert. Den Grund dafür suchte Euler in der falschen Bestimmung dessen, was unendlich kleine Größen sein sollen. Ausdrücklich gab er zu bedenken: ,Wie klein auch immer diese unendlich kleinen Größen aufgefasst werden, kann sich dennoch daraus schließlich ein gewaltiger Fehler ergeben, da nicht nur einzelne, sondern auch viele und sogar unendlich viele zugleich zu vernachlässigen sind“ (Euler 1775, S. 6).

Eulers radikale Lösung dieses Problems war, unendlich kleine Größen oder Differentiale müssen so gedeutet werden, dass sie überhaupt Null oder für Null gleich erachtet werden (Euler 1755, S. 5). „Wer fragt, was ein unendlich kleine Größe ist, dem antworten wir, dass sie in Wahrheit gleich Null ist". Diese Definition stimme damit überein, dass unendlich Kleines kleiner als jede zuordenbare Größe genannt werde (Euler 1755, S. 69).

Die letzte Bemerkung ist zwingend richtig: Euler musste mit Nullen rechnen, zwischen denen er aber Unterschiede machte. Aus

$$
2.0=1.0
$$

folgte für ihn $2: 1=0: 0$. Die Richtigkeit der Proportionalität setzte voraus, dass auf der rechten Gleichungsseite die linke Null größer war als die rechte Null sein müsste. Eine widerspruchsfreie Rechnung mit reellen Zahlen war so nicht zu begrün- 
den. In seinen Schriften zur Mechanik und Astronomie benötigte Euler unendlich kleine Größen, die ungleich Null waren. Dass man mit modernen Methoden der Nichtstandard-Analysis Eulers Umgang mit unendlich kleinen Größen deuten kann, ändert an diesem Befund nichts. Die richtige Lösung hatte Leibniz in Schriften gegeben, die zu Eulers Zeiten noch unveröffentlicht waren: „unendlich klein“ heißt „kleiner als jede zugeordnete Größ"“.

Euler war freilich überzeugt, die Kritiker des Differentialkalküls widerlegt zu haben. Stolz verkündete er (Euler 1755, S. 71): „Deshalb fällt jener Einwand, durch den die Analysis des Unendlichen beschuldigt wird, die geometrische Strenge zu vernachlässigen, von selbst zusammen. Denn es wird nichts anderes vernachlässigt als was in Wahrheit Null ist. Und deshalb kann man zu Recht behaupten, in dieser höheren Wissenschaft wird die höchst geometrische Strenge ebenso sorgfältig beachtet wie sie in den Büchern der Alten anzutreffen ist.“

\section{Zur Musiktheorie}

Seit alters her versuchte die Musiktheorie zu klären, welche Zahlen zur Bildung konsonanter Intervalle heranzuziehen waren, wo das Abgrenzungskriterium zwischen Konsonanz und Dissonanz zu suchen war. In einem berühmten Brief an Eulers späteren befreundeten Kollegen Christian Goldbach hatte Gottfried Wilhelm Leibniz 1712 geschrieben: „Wir zählen in der Musik nicht über Fünf hinaus, ähnlich jenen Völkern, die auch in der Arithmetik nicht über Drei fortschritten [...]. Denn alle unsere gebräuchlichen Intervalle gehören zu Verhältnissen, die aus je zwei der Primzahlen 1, 2, 3, 5 gebildet sind. Wenn uns ein wenig mehr Feinfühligkeit gegeben würde, könnten wir bis zur Primzahl 7 voranschreiten“ (Leibniz 1734, S. 240). Euler kannte diesen 1734 erstmals veröffentlichten Brief und kam darauf mehrfach zurück. Er wählte einen völlig neuen, rein mathematischen, zahlentheoretischen Zugang zur Lösung dieses Abgrenzungsproblems. In seinem Versuch einer Musiktheorie, die auf Grund der sichersten Prinzipien der Harmonie klar dargelegt ist, erklärte er seine Vorgehensweise (Euler 1739, S. 246): „Aber da es zum einen schwierig ist, die Grenzen der Konsonanzen und Dissonanzen zu bestimmen, zum anderen aber diese Unterscheidung mit unserer Behandlungsweise wenig übereinstimmt, [...] weisen wir allen Klängen, die aus mehreren gleichzeitig erklingenden Tönen bestehen, die Bezeichnung Konsonanz zu“". Mit anderen Worten: Euler hob die traditionelle Unterscheidung zwischen Konsonanzen und Dissonanzen auf.

Dazu definierte er zunächst für jede natürliche Zahl

$$
\mathrm{n}=p_{1}^{a_{1}} p_{2}{ }^{a_{2}} \ldots p_{m}{ }^{a_{m}}
$$

einen Annehmlichkeitsgrad (Euler 1739, S. 232f.) 
$\mathrm{G}(\mathrm{n})=\mathrm{a}_{1}\left(\mathrm{p}_{1}-1\right)+\ldots \mathrm{a}_{\mathrm{m}}\left(\mathrm{p}_{\mathrm{m}}-1\right)+1$

mit $\mathrm{G}(1):=1$.

Ist $\mathrm{n}=\mathrm{p}$ eine Primzahl, so gilt deshalb

$\mathrm{G}(\mathrm{p})=1(\mathrm{p}-1)+1=\mathrm{p}$

also Gleichheit. Es ist möglich, dass

$\mathrm{G}(\mathrm{n})=\mathrm{G}(\mathrm{m})$ für $\mathrm{n} \neq \mathrm{m}$

Die Funktion ist nicht injektiv. Als Beispiel diene

$\mathrm{n}=12=22.31 \mathrm{mit} \mathrm{G}(\mathrm{n})=2(2-1)+1(3-1)+1=5$

Intervallen bzw. Mehrklängen ordnete Euler als Konsonanzgrad den Annehmlichkeitsgrad des kleinsten gemeinsamen Vielfachen ihrer Verhältniszahlen zu:

$\mathrm{G}(\mathrm{a}: \mathrm{b})=\mathrm{G}(\mathrm{kgV}(\mathrm{a}, \mathrm{b}))$

Als Beispiele mögen dienen:

die große Terz 4:5 mit $\mathrm{kgV}(4,5)=20$ also mit dem Konsonanzgrad

$\mathrm{G}(20)=2(2-1)+1(5-1)+1=7$

der Durdreiklang $4: 5: 6$ mit $\mathrm{kgV}(4,5,6)=60$

also mit $\mathrm{G}(60)=9$

1. Intervalle mit kleineren Gradzahlen sind gefälliger und leichter verwendbar. Freilich führte Eulers Vorgehen auf zwei Probleme.

a) Der Begriff der Konsonanz ließ sich nicht in die Gradeinteilung einpassen (Vogel 1960, S. L), denn zum Beispiel Ganzton 9: 8, kleine Terz $6: 5$, kleine Sext $8: 5$ bekamen den gleichen Grad 8 zugewiesen.

Der Kritik Hugo Riemanns und Carl Stumpfs stehen zustimmende, tonpsychologische Urteile von Felix Auerbach und Hermann von Helmholtz gegenüber (Vogel 1960, S. LI).

b) Die Verwendung des kleinsten gemeinsamen Vielfachen $\mathrm{kgV}$ ließ Kürzungen bzw. Erweiterungen der Mehrklänge durch Weglassen oder Einschieben weiterer Töne zu, die in der musikalischen Praxis unzulässig sind:

Die Akkorde $\mathrm{c}$ e gis beziehungsweise 16:20:25 und c gis beziehungsweise 16:25 haben denselben Konsonanzgrad 13, da $\operatorname{kgV}(16,20,25)=\operatorname{kgV}(16,25)$.

Entsprechendes gilt von den Akkorden $\mathrm{c} \mathrm{h}, \mathrm{c} \mathrm{e} \mathrm{h,} \mathrm{g}$ h, $\mathrm{c} \mathrm{e} \mathrm{g}$ h beziehungsweise 8:15, 8:10:15, 8:12:15, 8:10:12:15 mit dem gemeinsamen Grad 10.

Der Akkord g h d f beziehungsweise 36:45:54:64 kann zur C-Dur-Tonleiter g a $\mathrm{h}$ c d e f beziehungsweise 36:40:45:48:54:60:64 mit dem gemeinsamen Grad 17 erweitert werden.

Aber nicht nur dissonante Mehrklänge kommen durch das Rechnen mit dem kgV ins Spiel. Es werden auch Akkord-Umkehrungen oder Tonverdopplungen nicht berücksichtigt. 
2. Der Wert des Systems liegt demgegenüber in zwei Grundannahmen dieser Konsonanzgradberechnung:

a) der Oktavabstufung

Eine Oktave mehr erhöht den Grad um 1:

$\mathrm{G}(2: 1)=2, \mathrm{G}(4: 1)=2(2-1)+1=3$

b) der Primzahleinordnung

Keine Primzahl ist mehr ausgeschlossen, auch die Sieben nicht. Euler trat für die Naturseptime 7:4 ein, um die hohe Konsonanz des Dominantseptimenakkords zu begründen. Erklärte er doch Musikgenuss mit Freude am Lösen eines (mathematischen) Rätsels (Euler 1768-1772, III, 11, S. 23f. [Brief 8]). Unter Bezugnahme auf die zitierte Stelle aus Leibnizens Brief bemerkte deshalb Euler 1766 scherzhaft (Euler 1766b, S. 525): „Alle diese Gründe verpflichten uns anzuerkennen, dass man auf die Primzahl 7 zurückgreifen muss, um den Erfolg dieser Akkorde zu erklären. Daher geht in die Verhältnisse, die die Natur dieser neuen Akkorde ausmachen, außer den Primzahlen 2, 3 und 5 noch die folgende 7 ein. Und mithin werden wir mit dem verstorbenen Herrn von Leibniz sagen können, dass die Musik gelernt hat, bis zur 7 zu zählen.“

\section{Epilog}

Eulers Glaube an die prinzipielle Lösbarkeit mathematischer Probleme erinnert an Hilberts Optimismus. Gegebenenfalls muss man die Probleme so fassen, dass sie lösbar werden. Wenn er ein Problem nicht lösen konnte, wie die algorithmische Lösung der algebraischen Gleichung höheren als 4. Grades (Thiele 1982, S. 103), wie das himmelsmechanische Dreikörperproblem, so suchte er die Gründe dafür in der unzureichenden Ausarbeitung des betreffenden Zweiges der Mathematik.

Um es mit den Worten Eduard Fueters aus dem Jahre 1941 zu sagen: „Denn wo der mathematische Verstand nicht hinreichte, begann für Euler das Reich Gottes“" (Fellmann 2007, S. 172).

\section{Literatur}

Die Werke Eulers werden nach dem Wiederabdruck in den Opera omnia (EO) zitiert, z. B. EO III, 12 = Reihe III, Band 12).

Die Übersetzungen der Zitate stammen, wenn nicht anders angegeben, vom Verfasser.

Breidert, Wolfgang (1983): Leonhard Euler und die Philosophie. In: Leonhard Euler 17071783, Beiträge zu Leben und Werk, Gedenkband des Kantons Basel-Stadt, Basel: Birkhäuser, S. 447-457. 
Condorcet, Marie Jean Antoine Nicolas Caritat, Marquis de (1786): Eloge de M. Euler. In: Histoire de l'Académie Royale des Sciences 1783, Paris 1786, S. 37-68 = EO III, 12, S. $287-310$

Engel, Friedrich \& Ludwig Schlesinger (1913): Vorwort der Herausgeber. In: EO I, 11, S. VII-XVIII.

Euler, Leonhard (1727): Dissertatio de sono, Basel: E. u. J. R. Thurneisen, Brüder = EO III, 1, S. 181-196.

Euler, Leonhard (1736): Mechanica sive motus scientia analytice exposita. 2 Bände, Petersburg: Academia Scientiarum = EO II, 1 und 2.

Euler, Leonhard (1739): Tentamen novae theoriae musicae ex certissimis harmoniae principiis dilucide expositae, Petersburg: Academia Scientiarum = EO III, 1, S. 197-427.

Euler, Leonhard (1740): De summis serierum reciprocarum. In: Commentarii academiae scientiarum Petropolitanae 7 (1734/35), 1740, S. 123-134 = EO I, 14, S. 73-86.

Euler, Leonhard (1743): De summis serierum reciprocarum ex potestatibus numerorum naturalium ortarum dissertation altera in qua eaedem summationes ex fonte maxime diverso derivantur. In: Miscellanea Berolinensia 7, S. 172-192 = EO I, 14, S. 138-155.

Euler, Leonhard (1744a.): Theoria motuum planetarum et cometarum. Continens methodum facilem ex aliquot observationibus orbitas cum planetarum tum cometarum determinandi. Una cum calculo, quo cometae, qui annis 1680 et 1681 itemque ejus, qui nuper est visus, motus verus investigatur, Berlin: A. Haude = EO II, 28, S. 105-268.

Euler, Leonhard (1744b.): Methodus inveniendi lineas curvas maximi minimive proprietate gaudentes, sive solutio problematis isoperimetrici latissimo sensu accepti, Lausanne-Genf: Marcus-Michaelis Bousquet = EO I, S. 24.

Euler, Leonhard (1745) : Neue Grundsätze der Artillerie enthaltend die Bestimmung der Gewalt des Pulvers nebst einer Untersuchung über den Unterschied des Wiederstands der Luft in schnellen und langsamen Bewegungen, aus dem Englischen des Hrn. Benjamin Robins übersetzt und mit den nöthigen Erläuterungen und vielen Anmerkungen versehen, Berlin: A. Haude = EO II, S. 14 .

Euler, Leonhard (1746a): Gedancken von den Elementen der Cörper, in welchen das LehrGebäude von den einfachen Dingen und Monaden geprüfet, und das wahre Wesen der Cörper entdecket wird, Berlin: A. Haude u. Joh. C. Spener = EO III, 2, S. 347-366.

Euler, Leonhard (1746b.): Enodatio quaestionis utrum materiae facultas cogitandi tribui possit necne ex principiis mechanicis petita. In: Opuscula varii argumenti 1, S. 277-286 = EO III, 2, S. 367-372.

Euler, Leonhard (1747): Rettung der Göttlichen Offenbahrung gegen die Einwürfe der Freygeister, Berlin: A. Haude u. J. C. Spener = EO III, 12, S. 267-372.

Euler, Leonhard (1748) : Introductio in analysin infinitorum, Lausanne: Marcus-Michaelis Bousquet $=$ EO I, S. 8 u. 9. 
Euler, Leonhard (1755): Institutiones calculi differentialis cum eius usu in analisi finitorum ac doctrina serierum, Berlin: (Auf Kosten der) Academia Imperialis Scientiarum.

Euler, Leonhard (1760): De seriebus divergentibus. In: Novi commentarii academiae scientiarum Petropolitanae 5 (1754/55), 1760, S. 205-237 = EO I, 14, S. 585-617.

Euler, Leonhard (1766a): Considerationes de motu corporum coelestium. In: Novi commentarii academiae scientiarum Petropolitanae 10 (1764), 1766, S. 544-558 = EO II, 25, S. 246-257.

Euler, Leonhard (1766b): Du véritable caractère de la musique moderne. In: Mémoires de l'académie des sciences de Berlin 20 (1764), 1766, S. 174-199= EO III, 1, S. 516-539.

Euler, Leonhard (1767) : Recherches générales sur la mortalité et la multiplication du genre humain. In: Mémoires de l'académie des sciences de Berlin 16 (1760), 1767, S. 144-164= EO I, 7, S. 79-100.

Euler, Leonhard (1768-1772): Lettres à une princesse d'Allemagne sur divers sujets de physique et de philosophie, St. Petersburg : Académie Impériale des Sciences = EO III, 11 u. 12.

Euler, Leonhard (1986): Briefe an eine deutsche Prinzessin über verschiedene Gegenstände der Physik und Philosophie. Aus dem Französischen übersetzt. Nachdruck der Ausgabe Leipzig 1769-1773. Eingeleitet und erläutert von Andreas Speiser. Mit einem Vorwort von Karin Reich, Braunschweig: Vieweg.

Faber, Georg (1935): Übersicht über die Bände 14, 15, 16, 16* der ersten Serie. In: EO I, 16, 2, S. VII-XCVI, CVI-CXII.

Fellmann, Emil A. (2007): Leonhard Euler, translated by Erika Gautschi and Walter Gautschi, Basel, Boston, Berlin: Birkhäuser.

Finster, Reinhard \& Gerd van den Henvel (1990): Gottfried Wilhelm Leibniz, Reinbek b. Hamburg: Rowohlt.

Grattan-Guinness, Ivor (1970): The development of the foundations of mathematical analysis from Euler to Riemann, Cambridge/Mass.: MIT Press.

Hoffmann, Utz (1992): Naturforscher, Ein Reiseführer zu Denkmälern und Sammlungen in Berlin und Brandenburg, Berlin: edition q.

Hofmann, Joseph Ehrenfried (1959): Um Eulers erste Reihenstudien. In: Sammelband der zu Ehren des 250. Geburtstages Leonhard Eulers der Deutschen Akademie der Wissenschaften zu Berlin vorgelegten Abhandlungen, hrsg. von Kurt Schröder, Berlin: AkademieVerlag, S. 139-208.

Kant, Immanuel (1755): Allgemeine Naturgeschichte und Theorie des Himmels, oder Versuch von der Verfassung und dem mechanischen Ursprunge des ganzen Weltgebäudes nach Newtonischen Grundsätzen abgehandelt, Königsberg, Leipzig: J. Fr. Petersen. - Ich zitiere den Wiederabdruck in: Immanuel Kant, Werke in sechs Bänden, hrsg. von Wilhelm Weischedel, Frankfurt am Main: Insel, 1960, Bd. I, S. 219-396. 
Kant, Immanuel (1784): Beantwortung der Frage: Was ist Aufklärung? In: Berlinische Monatsschrift, Dezember 1784, S. 481-494. - Ich zitiere den Wiederabdruck in: Immanuel Kant, Werke in sechs Bänden, hrsg. von Wilhelm Weischedel, Frankfurt am Main: Insel, 1964, Bd. VI, S. 51-61.

Knobloch, Eberhard (1995): Das große Spargesetz der Natur: Zur Tragikomödie zwischen Euler, Voltaire und Maupertuis. In: Mitteilungen der Deutschen Mathematiker-Vereinigung, Heft 3 (1995), S. 14-20.

Leibniz, Gottfried Wilhelm (1713): Epistola ad V. Cl. Christianum Wolfium, Professorem Matheseos Halensem, circa scientiam infiniti: Acta Eruditorum Suppl. 5, sect. 6, S. 264270. Wiederabgedruckt in: G. W. Leibniz, Mathematische Schriften hrsg. von C. I. Gerhardt. Bd. V, Halle: H. W. Schmidt, 1858, S. 382-387. (Nachdruck Hildesheim: Olms, 1962).

Leibniz, Gottfried Wilhelm (1734): Epistulae ad diversos theologici, iuridici, medici, philosophici, mathematici, historici, et philologici argumenti, hrsg. von Christian Kortholt. Bd. 1, Leipzig: B. Chr. Breitkopf.

Leibniz, Gottfried Wilhelm (1856): Mathematische Schriften, hrsg. v. C. I. Gerhardt. Bd. III, Halle: H. W. Schmidt (Nachdruck Hildesheim: Olms, 1962).

Polya, Georg (1954): Mathematik und plausibles Schliessen. Bd. 1 Induktion und Analogie in der Mathematik. Übersetzt von Lulu Bechtolsheim. 2. Aufl., Basel, Stuttgart: Birkhäuser.

Schiller, Friedrich (1794): Achter Brief über die ästhetische Erziehung des Menschen in einer Reihe von Briefen. - Ich zitiere den Wiederabdruck in: Friedrich Schiller, Sämtliche Werke, hrsg. von Gerhard Fricke und Herbert G. Göpfert, München: Hanser, 1960: Bd. V, S. 590-592.

Simmons, George F. (2007): Calculus gems, Brief lives and memorable mathematics. With portraits by Maceo Mitchell, Washington/DC: MAA.

Simmons, George F. (2007): Calculus gems, Brief lives and memorable Mathematics, Washington/DC: MAA.

Stäckel, Paul (1907/08): Eine vergessene Abhandlung Leonhard Eulers über die Summe der reziproken Quadrate der natürlichen Zahlen. In: Bibliotheca Mathematica 3. Folge 8, S. 37-54. - Ich zitiere den Wiederabdruck in: EO I, 14, S. 156-176.

Süssmilch, Johann Peter (1761): Die göttliche Ordnung in den Veränderungen des menschlichen Geschlechts, aus der Geburt, dem Tode und der Fortpflanzung desselben erwiesen. 2. Aufl., Berlin: Verlag des Buchladens der Realschule. - Ich zitiere die in Eulers Opera omnia - Ausgabe abgedruckten Abschnitte: EO I, 7, S. 507-534.

Szabó, István (1987): Geschichte der mechanischen Prinzipien und ihrer wichtigsten Anwendungen. 3. Aufl., Basel, Boston, Stuttgart: Birkhäuser.

Thiele, Rüdiger (1982): Leonhard Euler, Leipzig: Teubner.

Varadarajan, Veeravalli S. (2006): Euler through time: A new look at old themes, Providence: AMS.

Vogel, Martin (1960): Die Musikschriften Leonhard Eulers. In : EO III, 11, S. XLIV-LX. 